\title{
Robust Transmit Strategies for Multiantenna Bidirectional Broadcast Channels
}

\author{
Rafael F. Wyrembelski*, Tobias J. Oechtering ${ }^{\dagger}$, Holger Boche*, and Mikael Skoglund ${ }^{\dagger}$ \\ *Lehrstuhl für Theoretische Informationstechnik \\ Technische Universität München, Germany \\ ${ }^{\dagger}$ School of Electrical Engineering and the ACCESS Linnaeus Center \\ Royal Institute of Technology (KTH), Stockholm, Sweden
}

\begin{abstract}
In this work the multiantenna bidirectional broadcast channel under channel uncertainty is studied. This problem is motivated by the broadcast phase of the decode-and-forward bidirectional relaying protocol, where a relay node establishes a bidirectional communication between two other nodes while having only imperfect channel state information available. Different uncertainty models are investigated, where the nominal channels experience either a multiplicative or additive perturbation based on a spectral norm constraint. For these uncertainty models the corresponding capacity regions of the multiantenna bidirectional broadcast channel are analyzed. Further, robust transmit strategies are characterized and worst-case perturbations are identified.
\end{abstract}

\section{INTRODUCTION}

The concept of bidirectional relaying turns out to be a key technique to improve the performance of wireless networks such as ad-hoc, sensor, and even cellular systems. Accordingly, this is intensively discussed by the 3rd Generation Partnership Projects Long-Term Evolution Advanced (3GPP LTEAdvanced) group. Since a relay cannot transmit and receive at the same time and frequency, it needs orthogonal resources for transmission and reception. This can be done more efficiently, if bidirectional communication is considered [1-4]. In this work we consider bidirectional relaying in a threenode network, where a relay node establishes a bidirectional communication between two other nodes as shown in Figure 1. Furthermore, since spatial MIMO techniques can improve the performance significantly [5], we assume vector-valued transmission.

In the initial multiple access phase of a two-phase decodeand-forward protocol, the two nodes transmit their messages to the relay node which decodes them. This is the classical multiple access channel (MAC), which is well understood. Therefore, we concentrate on the succeeding broadcast phase in this work. Here, the relay re-encodes and transmits both messages in such a way that both receiving nodes can decode the other message using their own message from the previous phase as side information. This differs from the classical broadcast channel and is therefore known as bidirectional broadcast channel (BBC).

This work was partly supported by the German Research Foundation (DFG) under Grant BO 1734/25-1.

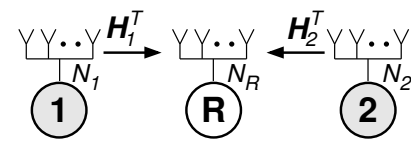

(a) MAC phase

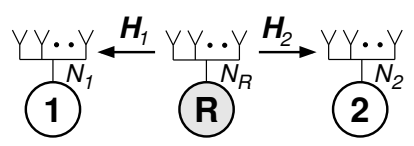

(b) BBC phase
Fig. 1. Decode-and-forward bidirectional relaying with multiple antennas.

The BBC is widely studied. Capacity achieving strategies can be found for instance in [6-9] for discrete memoryless channels and in [10] for MIMO Gaussian channels. Optimal transmit strategies for the multiantenna BBC are then analyzed in $[11,12]$. Besides the decode-and-forward protocol [6-15] there are also amplify-and-forward [15-19] or compress-andforward [20-22] approaches similarly as for the classical relay channel. A newer approach is compute-and-forward [23-29], where the relay decodes a certain function of both individual messages. Another approach is given in [30] which is based on the noisy network coding idea [31-33].

In practical systems there is always uncertainty in the channel state information (CSI) due to the nature of the wireless medium. But much more important is that CSI at the transmitter is usually realized by limited feedback schemes as for example in the current LTE system. This results in an inherent loss in available CSI. More precisely, the channel estimation is reported to the transmitter in terms of channel direction (CDI) and channel quality (CQI) information. The former specifies the beam from a pre-specified set of beamforming vectors while the latter characterizes a quantized SNR information corresponding to this beam. Especially the rough estimation of the channel quality necessitates the analysis of robust transmit strategies.

There are different approaches to tackle the problem of imperfect CSI at transmitter and receivers. On the one hand there are statistical approaches which assume the channel to be random but according to a certain statistic that is known. For example heuristics are developed for the multiantenna downlink scenario from a signal processing point of view in $[34,35]$. On the other hand there are deterministic models as the compound channel [36-38] where the actual channel is assumed to be fixed but it is only known that this channel belongs to a certain pre-specified set of channels. The corre- 
sponding MIMO compound channel is analyzed under various aspects in [39-42].

In this work we follow the deterministic uncertainty approach and therefore briefly revisit the discrete memoryless compound BBC in Section II. In Section III we introduce the uncertainty models which are considered for the multiantenna $\mathrm{BBC}$ in this work. We assume that the nominal channel experiences either a multiplicative or additive perturbation, where the set of possible perturbations is specified by a spectral norm constraint. Then we start with the analysis of the multiplicative uncertainty model for different antenna configurations in Section IV. Then we turn our attention to the additive uncertainty model in Section V. Finally, we end up with a conclusion in Section VI. ${ }^{1}$

\section{BidirectionAl BROAdCAST CHANNEL UNDER Channel UnCERTAinty ReVisited}

A reasonable way to model uncertainty in CSI is to assume that the actual channel realization over which the transmission takes place is unknown to the transmitter and the receivers. It is only known that this realization is from a pre-specified set of channels and that it remains fixed during the whole transmission of a codeword. This is the concept of compound channels [36-38] for which bidirectional relaying was analyzed for discrete memoryless channels in [43].

The discrete memoryless compound BBC is specified by an arbitrary (not necessarily finite) set of channels $\mathcal{S}$. Since transmitter and receivers only know the set $\mathcal{S}$ but not the actual realization $s \in \mathcal{S}$, a robust strategy is needed that works for all channel realizations in the set simultaneously. The corresponding compound capacity region is restated in the following theorem.

Theorem 1 ([43]): The compound capacity region of the discrete memoryless compound BBC is the set of all rate pairs $\left(R_{1}, R_{2}\right) \in \mathbb{R}_{+}^{2}$ that satisfy

$$
R_{1} \leq \inf _{s \in \mathcal{S}} I\left(\mathrm{X} ; \mathrm{Y}_{1, s}\right) \quad \text { and } \quad R_{2} \leq \inf _{s \in \mathcal{S}} I\left(\mathrm{X} ; \mathrm{Y}_{2, s}\right)
$$

for all random variables $\mathrm{X}$ and $\mathrm{Y}_{i, s}$ denoting the input of the relay node and the output at node $i, i=1,2$, for channel realization $s \in \mathcal{S}$.

Similarly as for the single-user compound channel [36, 37], the optimal input distributions are specified by max min optimization problems, since the transmitter does not know the actual channel realization and therefore has to choose the input distribution such that it works for all channel realizations simultaneously.

If there is channel state information at the transmitter (CSIT) available, the relay can adapt its input distribution according to the actual channel realization. Thus, the optimal

\footnotetext{
${ }^{1}$ Notation: Matrices, vectors, and sets are denoted by bold capital letters, bold lower case letters, and calligraphic letters; $\mathbb{C}$ and $\mathbb{R}_{+}$are the sets of complex and non-negative real numbers; $(\cdot)^{T}$ and $(\cdot)^{H}$ denote transpose and Hermitian transpose; $\arg (\cdot)$ denotes the phase of a complex number; $\operatorname{tr}(\cdot)$ and $\operatorname{rank}(\cdot)$ are the trace and the rank of a matrix; $\operatorname{diag}(\cdot)$ is a diagonal matrix and $\sigma_{k}(\cdot)$ is the $k$-th singular value of a matrix; $\boldsymbol{Q} \succeq \mathbf{0}$ means the matrix $\boldsymbol{Q}$ is positive semidefinite; $\mathbb{E}\{\cdot\}$ is the expectation; lhs := rhs assigns the right hand side (rhs) to the left hand side (lhs).
}

input distributions are specified by min max optimization problems. This kind of optimization is also called worst-case optimization. The corresponding worst-case capacity region of the compound $\mathrm{BBC}$ is restated in the following theorem.

Theorem 2 ([43]): The worst-case capacity region of the discrete memoryless compound $\mathrm{BBC}$ is the set of all rate pairs $\left(R_{1}, R_{2}\right) \in \mathbb{R}_{+}^{2}$ that satisfy

$$
R_{1} \leq \inf _{s \in \mathcal{S}} I\left(\mathrm{X}_{s} ; \mathrm{Y}_{1, s}\right) \quad \text { and } \quad R_{2} \leq \inf _{s \in \mathcal{S}} I\left(\mathrm{X}_{s} ; \mathrm{Y}_{2, s}\right)
$$

for all random variables $\mathrm{X}_{s}$ and $\mathrm{Y}_{i, s}, i=1,2$, where the input depends also on the channel realization $s \in \mathcal{S}$.

Remark 1: In general, the worst-case capacity is greater than the corresponding compound capacity which goes along with the intuition that available CSIT improves capacity. This is further confirmed by the observation that the solution of a min max optimization problem is always greater or equal than the solution of the corresponding max min optimization problem.

Remark 2: Although the compound BBC was only analyzed for discrete memoryless channels, we expect that the results given in Theorems 1 and 2 extend to MIMO Gaussian channels. We expect Gaussian input to be optimal so that the rates are given by well-known log det expressions. However, this should be explicitly analyzed from an optimal coding point of view and is left for future work.

\section{MOdeling THE UnCERTAINTY}

In this work we consider vector-valued transmission. Therefore we assume $N_{R}$ antennas at the relay node and $N_{i}$ antennas at node $i, i=1,2$, as shown in Figure 1. The input-output relation between the relay and node $i$ in the bidirectional broadcast (BBC) phase can be expressed as

$$
\boldsymbol{y}_{i}=\boldsymbol{x}^{T} \boldsymbol{H}_{i}+\boldsymbol{n}_{i}
$$

with $\boldsymbol{y}_{i} \in \mathbb{C}^{1 \times N_{i}}$ the output at node $i, \boldsymbol{H}_{i} \in \mathbb{C}^{N_{R} \times N_{i}}$ the multiplicative channel matrix, $\boldsymbol{x} \in \mathbb{C}^{N_{R} \times 1}$ the input of the relay node, and $\boldsymbol{n}_{i} \in \mathbb{C}^{1 \times N_{i}}$ the independent additive noise according to a circular symmetric complex Gaussian distribution $\mathcal{C} \mathcal{N}\left(\mathbf{0}, N \boldsymbol{I}_{N_{i}}\right), i=1,2$. We assume an average transmit power constraint $\operatorname{tr}(\boldsymbol{Q}) \leq P$ with $\boldsymbol{Q}=\mathbb{E}\left\{\boldsymbol{x} \boldsymbol{x}^{H}\right\}$.

We model the uncertainty in CSI by letting the channel $\boldsymbol{H}_{i}$, $i=1,2$, be consisting of two parts: a nominal part $\boldsymbol{H}_{i, 0}$ that is known to the relay and receiving node $i$, and the uncertainty (or perturbation) $\boldsymbol{D}_{i}$ that is unknown to both.

As in [40] we use the spectral (matrix) norm [44] to specify the uncertainty. We define the uncertainty set (or set of perturbations) for node $i$ as

$$
\mathcal{D}_{i}:=\left\{\boldsymbol{D}_{i}: \sigma_{1}\left(\boldsymbol{D}_{i}\right) \leq \epsilon_{i}\right\}
$$

where $\sigma_{k}\left(\boldsymbol{D}_{i}\right)$ denotes the $k$-th singular value of $\boldsymbol{D}_{i}, i=1,2$. The spectral norm has the advantage that it can be interpreted as the maximum transfer gain which perfectly matches to the uncertainty in channel quality (CQI) due to the quantized SNR information in practical systems. The use of the spectral norm has the additional advantage that it lower bounds any other 
matrix norm such as the Frobenius norm [44]. Thus, it further gives the largest uncertainty set $\mathcal{D}_{i}, i=1,2$.

We deal with two different kinds of uncertainty in this work: multiplicative and additive perturbation. In the multiplicative uncertainty model the nominal channel $\boldsymbol{H}_{i, 0}$ experiences a multiplicative perturbation so that

$$
\boldsymbol{H}_{i}=\boldsymbol{H}_{i, 0}\left(\boldsymbol{I}_{N_{i}}+\boldsymbol{D}_{i}\right)
$$

with $\boldsymbol{D}_{i} \in \mathbb{C}^{N_{i} \times N_{i}}$ or

$$
\boldsymbol{H}_{i}=\left(\boldsymbol{I}_{N_{R}}+\boldsymbol{D}_{i}\right) \boldsymbol{H}_{i, 0}
$$

with $\boldsymbol{D}_{i} \in \mathbb{C}^{N_{R} \times N_{R}}$ depending on if the nominal channel is multiplied by the uncertainty from the right or from the left. We identify (3) and (4) as receive-site and sent-site multiplicative uncertainty motivated by the common MIMO processing interpretation. For the multiplicative uncertainty model we additionally require $\sigma_{1}\left(\boldsymbol{D}_{i}\right) \leq \epsilon_{i}<1, i=1,2$, since otherwise the capacity region becomes empty.

In the additive uncertainty model the actual channel realization is given by

$$
\boldsymbol{H}_{i}=\boldsymbol{H}_{i, 0}+\boldsymbol{D}_{i}
$$

with $\boldsymbol{D}_{i} \in \mathbb{C}^{N_{R} \times N_{i}}, i=1,2$.

The goal is now to characterize what is at best possible for the multiantenna BBC under these different uncertainty models (3)-(5).

\section{Multiplicative UnCERTAinty}

In this section we analyze the bidirectional broadcast channel under multiplicative channel uncertainty.

\section{A. SIMO Bidirectional Broadcast Channel}

First, we look at the easiest case where the relay is equipped with a single antenna and the receiving nodes with multiple antennas. Then the input-output relation (1) simplifies to

$$
\boldsymbol{y}_{i}=x \boldsymbol{h}_{i}+\boldsymbol{n}_{i}, \quad i=1,2 .
$$

The difference to (1) is that the input is now a scalar and the channel matrix is a row vector which we denote by $\boldsymbol{h}_{i} \in$ $\mathbb{C}^{1 \times N_{i}}$ in this context.

Since the input of the relay node is scalar-valued, a Gaussian input distribution maximizes the rates of both links simultaneously regardless of the actual perturbation. Thus, the max min and min max optimization problems reveal a saddle-point property and the corresponding compound and worst-case capacity regions coincide. It remains to characterize the impact of the different uncertainty models.

In the case of receive-site uncertainty the uncertainty set $\mathcal{D}_{i}$ is given by (2). For sent-site uncertainty the perturbation $d_{i}$ is a scalar and the uncertainty set reduces to $\mathcal{D}_{i}=\left\{d_{i}: d_{i} \leq \epsilon_{i}\right\}$, $i=1,2$.

Theorem 3: The compound and worst-case capacity regions of the SIMO BBC under receive-site and sent-site multiplicative channel uncertainty (3) and (4) are given by all rate pairs $\left(R_{1}, R_{2}\right) \in \mathbb{R}_{+}^{2}$ that satisfy

$$
R_{i} \leq \log \left(1+\frac{P}{N}\left(1-\epsilon_{i}\right)^{2}\left\|\boldsymbol{h}_{i, 0}\right\|^{2}\right) .
$$

Moreover, the worst-case receive-site and sent-site perturbations are given by

$$
\boldsymbol{D}_{i}^{\mathrm{RX}}=-\epsilon_{i} \boldsymbol{I}_{N_{i}} \quad \text { and } \quad d_{i}^{\mathrm{TX}}=-\epsilon_{i} .
$$

Proof: For the receive-site uncertainty, (3) becomes $\boldsymbol{h}_{i}=$ $\boldsymbol{h}_{i, 0}\left(\boldsymbol{I}_{N_{i}}+\boldsymbol{D}_{i}\right)$ and we know from $[10,11]$ that for given $\boldsymbol{D}_{i}$ the rates are bounded by

$$
R_{i} \leq \log \left(1+\frac{P}{N}\left\|\boldsymbol{h}_{i, 0}\left(\boldsymbol{I}_{N_{i}}+\boldsymbol{D}_{i}\right)\right\|^{2}\right), \quad i=1,2 .
$$

Now we are interested in finding the minimum rates that are achievable for all perturbations $D_{i} \in \mathcal{D}_{i}$ simultaneously. Then the corresponding minimization problem is given by

$$
\begin{aligned}
& \min _{\boldsymbol{D}_{i}} \log \left(1+\frac{P}{N}\left\|\boldsymbol{h}_{i, 0}\left(\boldsymbol{I}_{N_{i}}+\boldsymbol{D}_{i}\right)\right\|^{2}\right) \\
\geq & \min _{\boldsymbol{D}_{i}} \log \left(1+\frac{P}{N}\left(\left\|\boldsymbol{h}_{i, 0}\right\|-\left\|\boldsymbol{h}_{i, 0} \boldsymbol{D}_{i}\right\|\right)^{2}\right) \\
\geq & \log \left(1+\frac{P}{N}\left(\left\|\boldsymbol{h}_{i, 0}\right\|-\epsilon_{i}\left\|\boldsymbol{h}_{i, 0}\right\|\right)^{2}\right) \\
= & \log \left(1+\frac{P}{N}\left(1-\epsilon_{i}\right)^{2}\left\|\boldsymbol{h}_{i, 0}\right\|^{2}\right)
\end{aligned}
$$

where the first inequality follows from the triangle inequality. Let the worst-case perturbation being $D_{i}^{\mathrm{RX}}=-\epsilon_{i} \boldsymbol{I}_{N_{i}}$, we see that we actually achieve the lower bound.

For sent-site uncertainty, (4) becomes $\boldsymbol{h}_{i}=\left(1+d_{i}\right) \boldsymbol{h}_{i, 0}$ so that the perturbation is scalar-valued. It is straightforward to show that the rates are given by (6) and that the worst-case perturbation is given by $d_{i}^{\mathrm{TX}}=-\epsilon_{i}, i=1,2$. The details are omitted for brevity.

\section{B. MISO Bidirectional Broadcast Channel}

Next, we study the more interesting case where the relay is equipped with multiple antennas and the receiving nodes each with only one antenna. The input-output relation (1) is now

$$
y_{i}=\boldsymbol{x}^{T} \boldsymbol{h}_{i}+n_{i}, \quad i=1,2 .
$$

The difference to (1) is that we now have single receive antennas so that the channel matrix is a column vector which we accordingly denote by $\boldsymbol{h}_{i} \in \mathbb{C}^{N_{R} \times 1}$.

In [11] we observed for perfect CSI that a single beam transmit strategy is optimal. This means that the optimal transmit covariance matrix $\boldsymbol{Q}=\mathbb{E}\left\{\boldsymbol{x} \boldsymbol{x}^{H}\right\}$ has rank one and can be expressed as $\boldsymbol{Q}=\boldsymbol{q} \boldsymbol{q}^{H}=P \boldsymbol{u}_{\boldsymbol{q}} \boldsymbol{u}_{\boldsymbol{q}}^{H}$. The corresponding capacity region is given by all rate pairs $\left(R_{1}, R_{2}\right) \in \mathbb{R}_{+}^{2}$ that satisfy

$$
R_{i}(\boldsymbol{Q}) \leq \log \left(1+\frac{P}{N}\left|\boldsymbol{h}_{i}^{H} \boldsymbol{u}_{\boldsymbol{q}}\right|^{2}\right)
$$

for all $\boldsymbol{Q}=P \boldsymbol{u}_{\boldsymbol{q}} \boldsymbol{u}_{\boldsymbol{q}}^{H}$ with $\operatorname{tr}(\boldsymbol{Q}) \leq P$.

This observation and the fact that current systems such as LTE usually apply a single beam strategy, cf. Section I, motivates us to focus especially on single beam transmit strategies in the following.

For multiplicative uncertainty we have to distinguish between receive-site and sent-site uncertainty. 


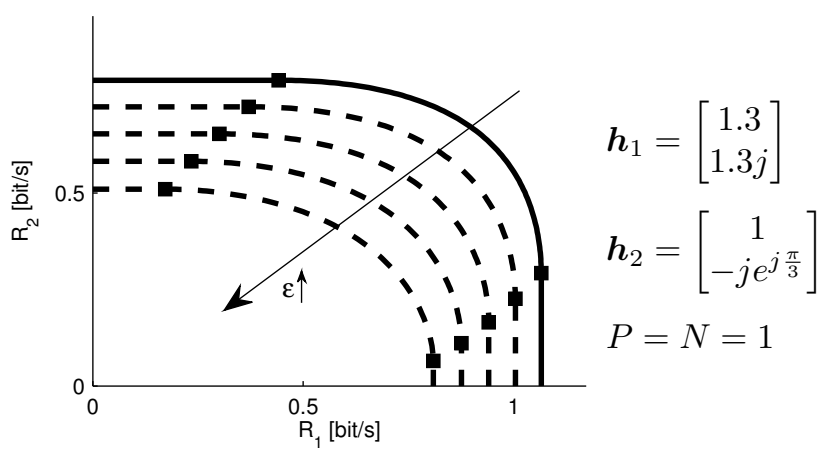

Fig. 2. Capacity region of the MISO BBC under receive-site multiplicative channel uncertainty for increasing $\epsilon=\epsilon_{1}=\epsilon_{2}$.

1) Receive-site uncertainty: This time, the perturbation $d_{i}$ is a scalar and the uncertainty set reduces to $\mathcal{D}_{i}=\left\{d_{i}: d_{i} \leq\right.$ $\left.\epsilon_{i}\right\}, i=1,2$. In this case we obtain the following result.

Theorem 4: The compound and worst-case capacity regions of the MISO BBC under receive-site multiplicative channel uncertainty (3) are given by all rate pairs $\left(R_{1}, R_{2}\right) \in \mathbb{R}_{+}^{2}$ that satisfy

$$
R_{i}(\boldsymbol{Q}) \leq \log \left(1+\frac{P}{N}\left(1-\epsilon_{i}\right)^{2}\left|\boldsymbol{h}_{i, 0}^{H} \boldsymbol{u}_{\boldsymbol{q}}\right|^{2}\right)
$$

for all $\boldsymbol{Q}=P \boldsymbol{u}_{\boldsymbol{q}} \boldsymbol{u}_{\boldsymbol{q}}^{H}, i=1,2$.

Proof: For given $\boldsymbol{h}_{i}=\boldsymbol{h}_{i, 0}\left(1+d_{i}\right)$ we know that the capacity region is given by

$$
\begin{aligned}
R_{i}(\boldsymbol{Q}) & \leq \log \left(1+\frac{P}{N}\left|\left(\boldsymbol{h}_{i, 0}\left(1+d_{i}\right)\right)^{H} \boldsymbol{u}_{\boldsymbol{q}}\right|^{2}\right) \\
& =\log \left(1+\frac{P}{N}\left(1+d_{i}\right)^{2}\left|\boldsymbol{h}_{i, 0}^{H} \boldsymbol{u}_{\boldsymbol{q}}\right|^{2}\right)
\end{aligned}
$$

for all $\boldsymbol{Q}=P \boldsymbol{u}_{\boldsymbol{q}} \boldsymbol{u}_{\boldsymbol{q}}^{H}$, cf. (7). Since the perturbation $d_{i}$ is scalar-valued, it is straightforward to show that $d_{i}^{\mathrm{RX}}=-\epsilon_{i}, i=$ 1,2 , and that the compound and worst-case capacity regions are characterized by (8) which completes the proof.

Interestingly, the worst-case perturbations do not depend on the transmit strategy $\boldsymbol{Q}$. Thus, the optimal single beam transmit strategy depends only the (known) nominal channels as in the case of perfect CSI.

Figure 2 depicts the loss in capacity due to the receivesite multiplicative channel uncertainty. As expected, with increasing uncertainty the capacity region shrinks.

2) Sent-site uncertainty: The scenario of sent-site uncertainty is much more involved than the previous one of receivesite uncertainty. We make the following observation by determining the worst-case rates for a given single beam transmit strategy.

Proposition 1: For given single beam transmit strategy $\boldsymbol{Q}=P \boldsymbol{u}_{\boldsymbol{q}} \boldsymbol{u}_{\boldsymbol{q}}^{H}$ the worst-case rates for the MISO BBC under sent-site multiplicative channel uncertainty (4) are given by

$$
R_{i}^{\mathrm{TX}}(\boldsymbol{Q})=\log \left(1+\frac{P}{N}\left(\left|\boldsymbol{h}_{i, 0}^{H} \boldsymbol{u}_{\boldsymbol{q}}\right|-\epsilon_{i}^{+}\left\|\boldsymbol{h}_{i, 0}\right\|\right)^{2}\right)
$$

with $\epsilon_{i}^{+}=\min \left\{\epsilon_{i},\left|\boldsymbol{h}_{i, 0}^{H} \boldsymbol{u}_{\boldsymbol{q}}\right| /\left\|\boldsymbol{h}_{i, 0}\right\|\right\}, i=1,2$. Moreover, the worst-case perturbation that yields this loss is given by

$$
\boldsymbol{D}_{i}^{\mathrm{TX}}(\boldsymbol{Q})=-\epsilon_{i} e^{-j \varphi_{i}} \boldsymbol{u}_{\boldsymbol{q}} \boldsymbol{u}_{i, 0}^{H}
$$

with $\varphi_{i}=\arg \left(\boldsymbol{h}_{i, 0}^{H} \boldsymbol{u}_{\boldsymbol{q}}\right)$.

Proof: From [11] we know that for given single beam transmit strategy $\boldsymbol{Q}=P \boldsymbol{u}_{\boldsymbol{q}} \boldsymbol{u}_{\boldsymbol{q}}^{H}$ the rates can be expressed as

$$
\begin{aligned}
& \min _{\boldsymbol{D}_{i}} \log \left(1+\frac{P}{N}\left|\boldsymbol{h}_{i, 0}^{H}\left(\boldsymbol{I}_{N_{R}}+\boldsymbol{D}_{i}\right)^{H} \boldsymbol{u}_{\boldsymbol{q}}\right|^{2}\right) \\
= & \min _{\boldsymbol{D}_{i}} \log \left(1+\frac{P}{N}\left|\boldsymbol{h}_{i, 0}^{H} \boldsymbol{u}_{\boldsymbol{q}}+\boldsymbol{h}_{i, 0}^{H} \boldsymbol{D}_{i}^{H} \boldsymbol{u}_{\boldsymbol{q}}\right|^{2}\right) \\
\geq & \min _{\boldsymbol{D}_{i}} \log \left(1+\frac{P}{N}\left(\left|\boldsymbol{h}_{i, 0}^{H} \boldsymbol{u}_{\boldsymbol{q}}\right|-\left|\boldsymbol{h}_{i, 0}^{H} \boldsymbol{D}_{i}^{H} \boldsymbol{u}_{\boldsymbol{q}}\right|\right)^{2}\right) \\
\geq & \log \left(1+\frac{P}{N}\left(\left|\boldsymbol{h}_{i, 0}^{H} \boldsymbol{u}_{\boldsymbol{q}}\right|-\epsilon_{i}\left\|\boldsymbol{h}_{i, 0}\right\|\right)^{2}\right)
\end{aligned}
$$

where the first inequality follows from the triangle inequality and the second inequality from

$$
\left|\boldsymbol{h}_{i, 0}^{H} \boldsymbol{D}_{i}^{H} \boldsymbol{u}_{\boldsymbol{q}}\right| \leq \epsilon_{i}\left\|\boldsymbol{h}_{i, 0}\right\|\left\|\boldsymbol{u}_{\boldsymbol{q}}\right\|=\epsilon_{i}\left\|\boldsymbol{h}_{i, 0}\right\| .
$$

Next, we have to show that this lower is actually achieved. Therefore, we choose $\boldsymbol{D}_{i}^{\mathrm{TX}}(\boldsymbol{Q})=-\epsilon_{i} e^{-j \varphi_{i}} \boldsymbol{u}_{\boldsymbol{q}} \boldsymbol{u}_{i, 0}^{H}$ with $\varphi_{i}=$ $\arg \left(\boldsymbol{h}_{i, 0}^{H} \boldsymbol{u}_{\boldsymbol{q}}\right)$ and obtain

$$
\begin{aligned}
R_{i}(\boldsymbol{Q}) & =\log \left(1+\frac{P}{N}\left|\boldsymbol{h}_{i, 0}^{H}\left(\boldsymbol{I}_{N_{R}}+\boldsymbol{D}_{i}^{\mathrm{TX}}(\boldsymbol{Q})\right)^{H} \boldsymbol{u}_{\boldsymbol{q}}\right|^{2}\right) \\
& =\log \left(1+\frac{P}{N}\left|\boldsymbol{h}_{i, 0}^{H} \boldsymbol{u}_{\boldsymbol{q}}-\epsilon_{i} e^{j \varphi_{i}} \boldsymbol{h}_{i, 0}^{H} \boldsymbol{u}_{i, 0} \boldsymbol{u}_{\boldsymbol{q}}^{H} \boldsymbol{u}_{\boldsymbol{q}}\right|^{2}\right) \\
& =\log \left(1+\frac{P}{N}|| \boldsymbol{h}_{i, 0}^{H} \boldsymbol{u}_{\boldsymbol{q}}\left|e^{j \varphi_{i}}-\epsilon_{i} e^{j \varphi_{i}}\left\|\boldsymbol{h}_{i, 0}\right\|\left\|\boldsymbol{u}_{\boldsymbol{q}}\right\|\right|^{2}\right) \\
& =\log \left(1+\frac{P}{N}|| \boldsymbol{h}_{i, 0}^{H} \boldsymbol{u}_{\boldsymbol{q}}\left|-\epsilon_{i}\left\|\boldsymbol{h}_{i, 0}\right\|\right|^{2}\right)=R_{i}^{\mathrm{TX}}(\boldsymbol{Q})
\end{aligned}
$$

which completes the proof.

An important observation in Proposition 1 is the following. In general, the worst-case sent-site perturbation $\boldsymbol{D}_{i}^{\mathrm{TX}}(\boldsymbol{Q})$ depends on the chosen transmit strategy $\boldsymbol{Q}$. Especially this means that if a single beam transmit strategy $\boldsymbol{Q}=P \boldsymbol{u}_{\boldsymbol{q}} \boldsymbol{u}_{\boldsymbol{q}}^{H}$ is given and fixed, the worst-case perturbation is immediately determined by $\boldsymbol{D}_{i}^{\mathrm{TX}}(\boldsymbol{Q})=-\epsilon_{i} e^{-j \varphi_{i}} \boldsymbol{u}_{\boldsymbol{q}} \boldsymbol{u}_{i, 0}^{H}$ depending on the given single beam direction $\boldsymbol{u}_{\boldsymbol{q}}$, cf. (9). On the other hand, we know from [11] that the optimal single beam transmit strategy itself depends on the actual channel realization $\boldsymbol{h}_{i}=\left(\boldsymbol{I}_{N_{R}}+\boldsymbol{D}_{i}\right) \boldsymbol{h}_{i, 0}$ and therewith on the perturbation $\boldsymbol{D}_{i}$, $i=1,2$.

This observation makes it very difficult to analyze the compound and worst-case capacity regions since they are determined by max min and min max optimization problems involving optimizations over both, the transmit strategy $Q$ and the perturbation $\boldsymbol{D}_{i}, i=1,2$. Moreover, note that a single beam transmit strategy need not be optimal anymore for maxmin optimization problems where the worst-case perturbation depends on the transmit strategy. Thus, it can be necessary to also characterize the worst-case perturbations for higher rank transmit strategies similarly as done for the single beam transmit strategy in Proposition 1, cf. (9).

\section{MIMO Bidirectional Broadcast Channel}

Here, we study the MIMO case where each node is equipped with multiple antennas. We consider receive-site uncertainty (3) and look at the worst-case rates for a given (not necessarily rank one) transmit strategy $\boldsymbol{Q}$. Basically, the following result follows immediately from [40, Lemma 1], but we present it for completeness in the following. 
Lemma 1 ([40]): For given transmit strategy $Q$ the worstcase rates for the MIMO BBC under receive-site multiplicative channel uncertainty (3) are given by

$$
R_{i}^{\mathrm{RX}}(\boldsymbol{Q})=\log \operatorname{det}\left(\boldsymbol{I}_{N_{i}}+\frac{1}{N}\left(1-\epsilon_{i}\right)^{2} \boldsymbol{H}_{i, 0}^{H} \boldsymbol{Q} \boldsymbol{H}_{i, 0}\right) .
$$

Moreover, the worst-case perturbation that yields this loss is given by

$$
\boldsymbol{D}_{i}^{\mathrm{RX}}(\boldsymbol{Q})=\boldsymbol{U}_{i, 0}^{\prime} \operatorname{diag}\left(-\epsilon_{i}\right) \boldsymbol{U}_{i, 0}^{\prime H},
$$

where $\boldsymbol{U}_{i, 0}^{\prime}$ comes from the singular value decomposition of the channel $\boldsymbol{H}_{i, 0}^{\prime}=\boldsymbol{H}_{i, 0}^{H} \boldsymbol{Q}^{1 / 2}$, i.e., $\boldsymbol{H}_{i, 0}^{\prime}=\boldsymbol{U}_{i, 0}^{\prime} \boldsymbol{\Sigma}_{i, 0}^{\prime} \boldsymbol{V}_{i, 0}^{\prime H}$, $i=1,2$.

Proof: For receive-site uncertainty we know from [10,12] that for given $\boldsymbol{D}_{i}$ the rates are bounded by

$$
R_{i}(\boldsymbol{Q}) \leq \log \operatorname{det}\left(\boldsymbol{I}_{N_{i}}+\frac{1}{N} \boldsymbol{H}_{i}^{H} \boldsymbol{Q} \boldsymbol{H}_{i}\right)
$$

with $\boldsymbol{H}_{i}=\boldsymbol{H}_{i, 0}\left(\boldsymbol{I}_{N_{i}}+\boldsymbol{D}_{i}\right), i=1,2$. Now we are interested in finding the minimum rates that are achievable for all possible perturbations $D_{i} \in \mathcal{D}_{i}$ simultaneously. Then the corresponding minimization problem is given by

$$
\begin{aligned}
& \min _{\boldsymbol{D}_{i}} \log \operatorname{det}\left(\boldsymbol{I}_{N_{i}}+\frac{1}{N} \boldsymbol{H}_{i}^{H} \boldsymbol{Q} \boldsymbol{H}_{i}\right) \\
= & \min _{\boldsymbol{D}_{i}} \sum_{k=1}^{n} \log \left(1+\frac{1}{N} \sigma_{k}^{2}\left(\boldsymbol{H}_{i}^{H} \boldsymbol{Q}^{1 / 2}\right)\right) \\
\geq & \sum_{k=1}^{n} \log \left(1+\frac{1}{N}\left(1-\epsilon_{i}\right)^{2} \sigma_{k}^{2}\left(\boldsymbol{H}_{i, 0}^{H} \boldsymbol{Q}^{1 / 2}\right)\right) \\
= & \log \operatorname{det}\left(\boldsymbol{I}_{N_{i}}+\frac{1}{N}\left(1-\epsilon_{i}\right)^{2} \boldsymbol{H}_{i, 0}^{H} \boldsymbol{Q} \boldsymbol{H}_{i, 0}\right) .
\end{aligned}
$$

The inequality follows from

$$
\begin{aligned}
\sigma_{k}^{2}\left(\boldsymbol{H}_{i}^{H} \boldsymbol{Q}^{1 / 2}\right) & =\left(\sigma_{k}\left(\left(\boldsymbol{I}_{N_{i}}+\boldsymbol{D}_{i}\right)^{H} \boldsymbol{H}_{i, 0}^{H} \boldsymbol{Q}^{1 / 2}\right)\right)^{2} \\
& \geq\left(\sigma_{n}\left(\boldsymbol{I}_{N_{i}}+\boldsymbol{D}_{i}^{H}\right) \sigma_{k}\left(\boldsymbol{H}_{i, 0}^{H} \boldsymbol{Q}^{1 / 2}\right)\right)^{2} \\
& \geq\left(\left(\sigma_{n}\left(\boldsymbol{I}_{N_{i}}\right)-\sigma_{1}\left(\boldsymbol{D}_{i}^{H}\right)\right) \sigma_{k}\left(\boldsymbol{H}_{i, 0}^{H} \boldsymbol{Q}^{1 / 2}\right)\right)^{2} \\
& \geq\left(\left(1-\epsilon_{i}\right) \sigma_{k}\left(\boldsymbol{H}_{i, 0}^{H} \boldsymbol{Q}^{1 / 2}\right)\right)^{2} \\
& =\left(1-\epsilon_{i}\right)^{2} \sigma_{k}^{2}\left(\boldsymbol{H}_{i, 0}^{H} \boldsymbol{Q}^{1 / 2}\right)
\end{aligned}
$$

where we used the relations $\sigma_{n}(\boldsymbol{A}) \sigma_{k}(\boldsymbol{B}) \leq \sigma_{k}(\boldsymbol{A} \boldsymbol{B})$ and $\sigma_{k}(\boldsymbol{B})-\sigma_{1}(\boldsymbol{C}) \leq \sigma_{k}(\boldsymbol{B}+\boldsymbol{C}), k=\min \left\{N_{i}, N_{R}\right\}$ with $\boldsymbol{A} \in$ $\mathbb{C}^{N_{i} \times N_{i}}$ and $\boldsymbol{B}, \boldsymbol{C} \in \mathbb{C}^{N_{i} \times N_{R}}$, cf. also [40,44].

It remains to check that this lower bound is achieved. Therefore we choose $\boldsymbol{D}_{i}^{\mathrm{RX}}(\boldsymbol{Q})=\boldsymbol{U}_{i, 0}^{\prime} \operatorname{diag}\left(-\epsilon_{i}\right) \boldsymbol{U}_{i, 0}^{\prime H}$ to obtain the desired rates which completes the proof.

Unfortunately, we are again in the situation that the worstcase perturbations as given in (10) depend on the transmit strategy. But again, the optimal transmit strategy itself depend on the channel realization and therewith on the actual perturbation. Thus, the analysis of the compound and worst-case capacity regions becomes a hard task.

Therefore we consider in the following the special case of parallel nominal channels. Accordingly, as in [12, Sec. IV] let

$$
\boldsymbol{H}_{i, 0} \boldsymbol{H}_{i, 0}^{H}=\boldsymbol{W}_{i} \boldsymbol{S}_{i} \boldsymbol{W}_{i}^{H}
$$

with $\boldsymbol{S}_{i}=\operatorname{diag}\left(s_{i, 1}, s_{i, 2}, \ldots, s_{i, n}\right) \succeq \mathbf{0}$ denote the eigenvalue decomposition of each channel. Then for parallel channels we assume that the unitary matrices $\boldsymbol{W}_{1}$ and $\boldsymbol{W}_{2}$ are equal, i.e., we have $\boldsymbol{W}=\boldsymbol{W}_{1}=\boldsymbol{W}_{2}$. For parallel channels we know from [12, Proposition 3] that the optimal transmit covariance matrix $Q$ has the eigenvalue decomposition

$$
\boldsymbol{Q}=\boldsymbol{W} \boldsymbol{\Sigma}_{\boldsymbol{Q}} \boldsymbol{W}^{H}
$$

with $\boldsymbol{\Sigma}_{\boldsymbol{Q}}=\operatorname{diag}\left(\lambda_{1}, \lambda_{2}, \ldots, \lambda_{N_{R}}\right) \succeq \mathbf{0}$.

With the result we can analyze the compound and worstcase capacity regions for the special case of parallel channels in more detail.

Theorem 5: The compound capacity region of the parallel MIMO BBC with receive-site multiplicative channel uncertainty (3) is given by all rate pairs $\left(R_{1}, R_{2}\right) \in \mathbb{R}_{+}^{2}$ that satisfy

$$
R_{i}(\boldsymbol{Q}) \leq \log \operatorname{det}\left(\boldsymbol{I}_{N_{i}}+\frac{1}{N}\left(1-\epsilon_{i}\right)^{2} \boldsymbol{S}_{i} \boldsymbol{\Sigma}_{\boldsymbol{Q}}\right)
$$

for all power allocations $\boldsymbol{\Sigma}_{\boldsymbol{Q}}=\operatorname{diag}\left(\lambda_{1}, \lambda_{2}, \ldots, \lambda_{N_{R}}\right) \succeq \mathbf{0}$.

Proof: Since the compound capacity region is convex, we can characterize it by their boundary which corresponds to the set of weighted rate sum optimal rate pairs. The weighted rate sum for a given weight vector $\boldsymbol{w}=\left(w_{1}, w_{2}\right) \in \mathbb{R}_{+}^{2}$ with $w_{1}+w_{2}=1$, given transmit covariance matrix $\boldsymbol{Q}$, and given perturbation $\boldsymbol{D}=\left(\boldsymbol{D}_{1}, \boldsymbol{D}_{2}\right)$ is given by

$$
R_{\Sigma}(\boldsymbol{w}, \boldsymbol{Q}, \boldsymbol{D}):=\sum_{i=1}^{2} w_{i} \log \operatorname{det}\left(\boldsymbol{I}_{N_{i}}+\frac{1}{N} \boldsymbol{H}_{i}^{H} \boldsymbol{Q} \boldsymbol{H}_{i}\right) .
$$

Then the boundary is characterized by max min optimization problems as

$$
\begin{aligned}
& R_{\Sigma, \max m i n}(\boldsymbol{w})=\max _{\boldsymbol{Q}} \min _{\boldsymbol{D}} R_{\Sigma}(\boldsymbol{w}, \boldsymbol{Q}, \boldsymbol{D}) \\
& \quad=\max _{\boldsymbol{Q}} \sum_{i=1}^{2} w_{i} \min _{\boldsymbol{D}_{i}} \log \operatorname{det}\left(\boldsymbol{I}_{N_{i}}+\frac{1}{N} \boldsymbol{H}_{i}^{H} \boldsymbol{Q} \boldsymbol{H}_{i}\right) \\
& \quad=\max _{\boldsymbol{Q}} \sum_{i=1}^{2} w_{i} \log \operatorname{det}\left(\boldsymbol{I}_{N_{i}}+\frac{1}{N}\left(1-\epsilon_{i}\right)^{2} \boldsymbol{H}_{i, 0}^{H} \boldsymbol{Q} \boldsymbol{H}_{i, 0}\right) \\
& \quad=\max _{\Sigma_{\boldsymbol{Q}}} \sum_{i=1}^{2} w_{i} \log \operatorname{det}\left(\boldsymbol{I}_{N_{i}}+\frac{1}{N}\left(1-\epsilon_{i}\right)^{2} \boldsymbol{S}_{i} \boldsymbol{\Sigma}_{\boldsymbol{Q}}\right)
\end{aligned}
$$

where the second last equality follows from Lemma 1 and the last equality from the assumption of parallel channels and the decomposition (11).

We observe that it is always optimal to transmit into the direction of the nominal channels as it is optimal for the point-to-point MIMO channel. Since the directions of the nominal channel and the transmit strategy coincide, the worstcase perturbation depends only on the channel direction. This makes it possible to characterize the compound capacity region as done in Theorem 5. Next, we look at the corresponding worst-case capacity region.

Theorem 6: The worst-case capacity region of the parallel MIMO BBC with receive-site multiplicative channel uncertainty (3) equals the corresponding compound capacity region of Theorem 5 and is given by all rate pairs $\left(R_{1}, R_{2}\right) \in \mathbb{R}_{+}^{2}$ that satisfy

$$
R_{i}(\boldsymbol{Q}) \leq \log \operatorname{det}\left(\boldsymbol{I}_{N_{i}}+\frac{1}{N}\left(1-\epsilon_{i}\right)^{2} \boldsymbol{S}_{i} \boldsymbol{\Sigma}_{\boldsymbol{Q}}\right)
$$


for all power allocations $\boldsymbol{\Sigma}_{\boldsymbol{Q}}=\operatorname{diag}\left(\lambda_{1}, \lambda_{2}, \ldots, \lambda_{N_{R}}\right) \succeq \mathbf{0}$.

Proof: Since the compound and worst-case capacity regions are both convex, we can characterize them by their boundaries. As in the proof of Theorem 5 we define the weighted rate sum for a given weight vector $\boldsymbol{w}=\left(w_{1}, w_{2}\right) \in$ $\mathbb{R}_{+}^{2}$ with $w_{1}+w_{2}=1$, given transmit covariance matrix $\boldsymbol{Q}$, and given perturbation $\boldsymbol{D}=\left(\boldsymbol{D}_{1}, \boldsymbol{D}_{2}\right)$ as

$$
R_{\Sigma}(\boldsymbol{w}, \boldsymbol{Q}, \boldsymbol{D}):=\sum_{i=1}^{2} \log \operatorname{det}\left(\boldsymbol{I}_{N_{i}}+\frac{1}{N} \boldsymbol{H}_{i}^{H} \boldsymbol{Q} \boldsymbol{H}_{i}\right) .
$$

Then the boundaries of the compound and worst-case capacity regions are characterized by max min and min max optimization problems as

$$
R_{\Sigma, \operatorname{maxmin}}(\boldsymbol{w})=\max _{\boldsymbol{Q}} \min _{\boldsymbol{D}} R_{\Sigma}(\boldsymbol{w}, \boldsymbol{Q}, \boldsymbol{D})
$$

and

$$
R_{\Sigma, \operatorname{minmax}}(\boldsymbol{w})=\min _{\boldsymbol{D}} \max _{\boldsymbol{Q}} R_{\Sigma}(\boldsymbol{w}, \boldsymbol{Q}, \boldsymbol{D})
$$

respectively. Note that the relation

$$
R_{\Sigma, \operatorname{minmax}}(\boldsymbol{w}) \geq R_{\Sigma, \operatorname{maxmin}}(\boldsymbol{w})
$$

always holds for all weight vectors $\boldsymbol{w} \in \mathbb{R}_{+}^{2}$ so that it remains to show the reversed inclusion to prove equality of both capacity regions. To do this we choose a specific perturbation $\boldsymbol{D}_{i}=-\epsilon_{i} \boldsymbol{I}, i=1,2$, and obtain for every weight vector $\boldsymbol{w}$

$$
\begin{aligned}
& R_{\Sigma, \operatorname{minmax}}(\boldsymbol{w}) \\
& \quad=\min _{\boldsymbol{D}} \max _{\boldsymbol{Q}} \sum_{i=1}^{2} \log \operatorname{det}\left(\boldsymbol{I}_{N_{i}}+\frac{1}{N} \boldsymbol{H}_{i}^{H} \boldsymbol{Q} \boldsymbol{H}_{i}\right) \\
& \quad \leq \max _{\boldsymbol{Q}} \sum_{i=1}^{2} \log \operatorname{det}\left(\boldsymbol{I}_{N_{i}}+\frac{1}{N}\left(1-\epsilon_{i}\right)^{2} \boldsymbol{H}_{i, 0}^{H} \boldsymbol{Q} \boldsymbol{H}_{i, 0}\right) \\
& \quad=R_{\Sigma, \max \min }(\boldsymbol{w})
\end{aligned}
$$

where the inequality follows from the simple observation that a specific perturbation is always greater or equal than the minimum over all possible perturbations. This completes the proof.

The optimal transmit directions are independent of the weight vector $\boldsymbol{w}$ so that it remains to determine the optimal power allocation allocation between the different beams for certain weights. Analogously to [12, Sec. IV] this can be expressed by the following optimization problem

$$
\begin{gathered}
R_{\Sigma, \operatorname{maxmin}}(\boldsymbol{w})=\max _{\boldsymbol{\Lambda}} \sum_{i=1}^{2} \sum_{k=1}^{N_{R}} w_{i} \log \left(1+\frac{1}{N}\left(1-\epsilon_{i}\right)^{2} s_{i, k}, \lambda_{k}\right) \\
\text { s.t. } \quad\|\boldsymbol{\lambda}\| \leq P, \lambda_{k} \geq 0, k=1,2, \ldots, N_{R}
\end{gathered}
$$

with $\boldsymbol{\lambda}=\left(\lambda_{1}, \lambda_{2}, \ldots, \lambda_{N_{R}}\right)$. If we compare this to the case of perfect CSI in [12, Sec. IV], we see that the optimization problem only differs in the factor $\left(1-\epsilon_{i}\right)^{2}$ in the objective function. Thus, the optimal power allocation can easily be deduced from [12, Sec. IV].

Remark 3: Here we considered vector-valued transmission in the spatial domain, but the results obviously also apply to

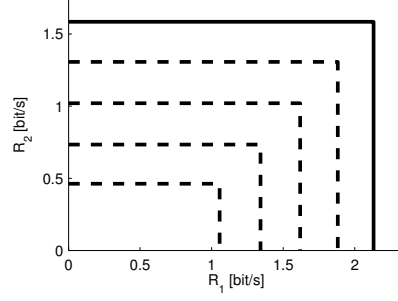

(a) Additive Uncertainty

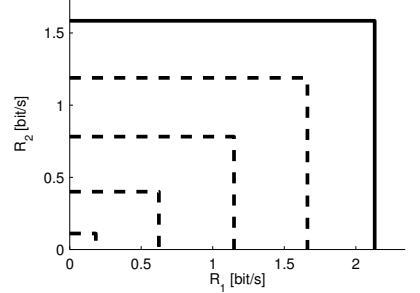

(b) Multiplicative Uncertainty
Fig. 3. Capacity regions of the SIMO BBC under additive and multiplicative uncertainty for equidistantly increasing $\epsilon=\epsilon_{1}=\epsilon_{2}$ for $\boldsymbol{h}_{1}=\left[\begin{array}{ll}1.3 & 1.3 j\end{array}\right]$, $\boldsymbol{h}_{2}=\left[\begin{array}{ll}1 & -j e^{-j \pi / 3}\end{array}\right]$, and $N_{R}=1, N_{1}=N_{2}=2$.

vector-valued processing in the frequency domain. Especially the scenario of parallel MIMO Gaussian channels matches a single-antenna OFDM system where the unitary matrix $W$ equals the IDFT-matrix. Thus, these results also characterize the optimal power allocation for single-antenna OFDM systems.

\section{AdDitive UnCERTAINTY}

In this section we analyze the bidirectional broadcast channel under additive channel uncertainty.

\section{A. SIMO Bidirectional Broadcast Channel}

Similarly to the channel vector, the additive perturbation is also a row vector which we denote by $\boldsymbol{d}_{i} \in \mathbb{C}^{1 \times N_{i}}, i=1,2$. The corresponding uncertainty set (2) becomes

$$
\mathcal{D}_{i}:=\left\{\boldsymbol{d}_{i}: \sigma_{1}\left(\boldsymbol{d}_{i}\right)=\left\|\boldsymbol{d}_{i}\right\| \leq \epsilon_{i}\right\} .
$$

Theorem 7: The compound and worst-case capacity regions of the SIMO BBC under additive channel uncertainty (5) are given by all rate pairs $\left(R_{1}, R_{2}\right) \in \mathbb{R}_{+}^{2}$ that satisfy

$$
R_{i} \leq \log \left(1+\frac{P}{N}\left(\left\|\boldsymbol{h}_{i, 0}\right\|-\epsilon_{i}^{+}\right)^{2}\right)
$$

with $\epsilon_{i}^{+}=\min \left\{\epsilon_{i},\left\|\boldsymbol{h}_{i, 0}\right\|\right\}, i=1,2$. Moreover, the worst-case additive perturbation that yields this loss is given by

$$
\boldsymbol{d}_{i}^{\text {add }}=-\epsilon_{i} \boldsymbol{u}_{i, 0}
$$

with $\boldsymbol{u}_{i, 0}=\boldsymbol{h}_{i, 0} /\left\|\boldsymbol{h}_{i, 0}\right\|, i=1,2$, the direction of the nominal channel.

Proof: From [10,11] we know that for any channel realization $\boldsymbol{h}_{i}=\boldsymbol{h}_{i, 0}+\boldsymbol{d}_{i}$, the rates are bounded by

$$
R_{i} \leq \log \left(1+\frac{P}{N}\left\|\boldsymbol{h}_{i, 0}+\boldsymbol{d}_{i}\right\|^{2}\right), \quad i=1,2 .
$$

Now we are interested in finding the minimum rates that are achievable for all perturbations $\boldsymbol{d}_{i} \in \mathcal{D}_{i}$ simultaneously, cf. (12). We have

$$
\begin{aligned}
& \min _{\boldsymbol{d}_{i}} \log \left(1+\frac{P}{N}\left\|\boldsymbol{h}_{i, 0}+\boldsymbol{d}_{i}\right\|^{2}\right) \\
\geq & \min _{\boldsymbol{d}_{i}} \log \left(1+\frac{P}{N}\left(\left\|\boldsymbol{h}_{i, 0}\right\|-\left\|\boldsymbol{d}_{i}\right\|\right)^{2}\right) \\
= & \log \left(1+\frac{P}{N}\left(\left\|\boldsymbol{h}_{i, 0}\right\|-\epsilon_{i}\right)^{2}\right)
\end{aligned}
$$

where the inequality follows from the triangle inequality. To complete the proof it remains to show that this lower bound is 
tight. Therefore we choose the worst-case perturbation $\boldsymbol{d}_{i}^{\text {add }}=$ $-\epsilon_{i} \boldsymbol{u}_{i, 0}$, cf. (13), which actually yields the desired rates.

As expected Theorem 7 shows that the additive worst-case perturbation is anti-parallel to the nominal channel so that the gain of the nominal channel is reduced most.

Figure 3 depicts the capacity regions of the SIMO BBC under additive and multiplicative uncertainty. It shows the different influence of increasing uncertainty $\epsilon$.

\section{B. MISO Bidirectional Broadcast Channel}

As in the corresponding SIMO case in the previous subsection, the additive perturbation is a vector $\boldsymbol{d}_{i} \in \mathbb{C}^{N_{R} \times 1}$, $i=1,2$, and the uncertainty set is given by (12). Similarly as for sent-site multiplicative uncertainty, cf. Section IV-B, we determine the worst-case rates for a given single beam transmit strategy.

Proposition 2: For given single beam transmit strategy $\boldsymbol{Q}=P \boldsymbol{u}_{\boldsymbol{q}} \boldsymbol{u}_{\boldsymbol{q}}^{H}$ the worst-case rates for the MISO BBC under additive channel uncertainty (5) are given by

$$
R_{i}^{\text {add }}(\boldsymbol{Q})=\log \left(1+\frac{P}{N}\left(\left|\boldsymbol{h}_{i, 0}^{H} \boldsymbol{u}_{\boldsymbol{q}}\right|-\epsilon_{i}^{+}\right)^{2}\right)
$$

with $\epsilon_{i}^{+}=\min \left\{\epsilon_{i},\left|\boldsymbol{h}_{i, 0}^{H} \boldsymbol{u}_{\boldsymbol{q}}\right|\right\}, i=1,2$. Moreover, the worstcase perturbation that yields this loss is given by

$$
\boldsymbol{d}_{i}^{\text {add }}(\boldsymbol{Q})=-\epsilon_{i} e^{-j \varphi_{i}} \boldsymbol{u}_{\boldsymbol{q}}
$$

with $\varphi_{i}=\arg \left(\boldsymbol{h}_{i, 0}^{H} \boldsymbol{u}_{\boldsymbol{q}}\right), i=1,2$.

Proof: From [11] we know that for given single beam transmit strategy $\boldsymbol{Q}=P \boldsymbol{u}_{\boldsymbol{q}} \boldsymbol{u}_{\boldsymbol{q}}^{H}$ and $\boldsymbol{h}_{i}=\boldsymbol{h}_{i, 0}+\boldsymbol{d}_{i}$, the rates can be expressed as (7). Now we are interested in finding the minimum rates that are achievable for all perturbations $\boldsymbol{d}_{i} \in \mathcal{D}_{i}$ simultaneously. We have

$$
\begin{aligned}
& \min _{\boldsymbol{d}_{i}} \log \left(1+\frac{P}{N}\left|\left(\boldsymbol{h}_{i, 0}+\boldsymbol{d}_{i}\right)^{H} \boldsymbol{u}_{\boldsymbol{q}}\right|^{2}\right) \\
= & \min _{\boldsymbol{d}_{i}} \log \left(1+\frac{P}{N}\left|\boldsymbol{h}_{i, 0}^{H} \boldsymbol{u}_{\boldsymbol{q}}+\boldsymbol{d}_{i}^{H} \boldsymbol{u}_{\boldsymbol{q}}\right|^{2}\right) \\
\geq & \min _{\boldsymbol{d}_{i}} \log \left(1+\frac{P}{N}\left(\left|\boldsymbol{h}_{i, 0}^{H} \boldsymbol{u}_{\boldsymbol{q}}\right|-\left|\boldsymbol{d}_{i}^{H} \boldsymbol{u}_{\boldsymbol{q}}\right|\right)^{2}\right) \\
\geq & \log \left(1+\frac{P}{N}\left(\left|\boldsymbol{h}_{i, 0}^{H} \boldsymbol{u}_{\boldsymbol{q}}\right|-\epsilon_{i}\right)^{2}\right)
\end{aligned}
$$

where the first inequality follows from the triangle inequality and the second inequality from

$$
\left|\boldsymbol{d}_{i}^{H} \boldsymbol{u}_{\boldsymbol{q}}\right| \leq\left\|\boldsymbol{d}_{i}\right\|\left\|\boldsymbol{u}_{\boldsymbol{q}}\right\|=\left\|\boldsymbol{d}_{i}\right\| \leq \epsilon_{i}
$$

Next, we have to show that this lower bound is tight. Therefore, we choose $\boldsymbol{d}_{i}^{\text {add }}(\boldsymbol{Q})=-\epsilon_{i} e^{-j \varphi_{i}} \boldsymbol{u}_{\boldsymbol{q}}$ with $\varphi_{i}=$ $\arg \left(\boldsymbol{h}_{i, 0}^{H} \boldsymbol{u}_{\boldsymbol{q}}\right)$ and obtain

$$
\begin{aligned}
R_{i}(\boldsymbol{Q}) & =\log \left(1+\frac{P}{N}\left|\left(\boldsymbol{h}_{i, 0}+\boldsymbol{d}_{i}^{\mathrm{add}}(\boldsymbol{Q})\right)^{H} \boldsymbol{u}_{\boldsymbol{q}}\right|^{2}\right) \\
& =\log \left(1+\frac{P}{N}\left|\left(\boldsymbol{h}_{i, 0}-\epsilon_{i} e^{-j \varphi_{i}} \boldsymbol{u}_{\boldsymbol{q}}\right)^{H} \boldsymbol{u}_{\boldsymbol{q}}\right|^{2}\right) \\
& =\log \left(1+\frac{P}{N}\left|\boldsymbol{h}_{i, 0}^{H} \boldsymbol{u}_{\boldsymbol{q}}-\epsilon_{i} e^{j \varphi_{i}} \boldsymbol{u}_{\boldsymbol{q}}^{H} \boldsymbol{u}_{\boldsymbol{q}}\right|^{2}\right) \\
& =\log \left(1+\frac{P}{N}|| \boldsymbol{h}_{i, 0}^{H} \boldsymbol{u}_{\boldsymbol{q}}\left|e^{j \varphi_{i}}-\epsilon_{i} e^{j \varphi_{i}}\right|^{2}\right) \\
& =\log \left(1+\frac{P}{N}\left(\left|\boldsymbol{h}_{i, 0}^{H} \boldsymbol{u}_{\boldsymbol{q}}\right|-\epsilon_{i}\right)^{2}\right) \\
& =R_{i}^{\mathrm{add}}(\boldsymbol{Q})
\end{aligned}
$$

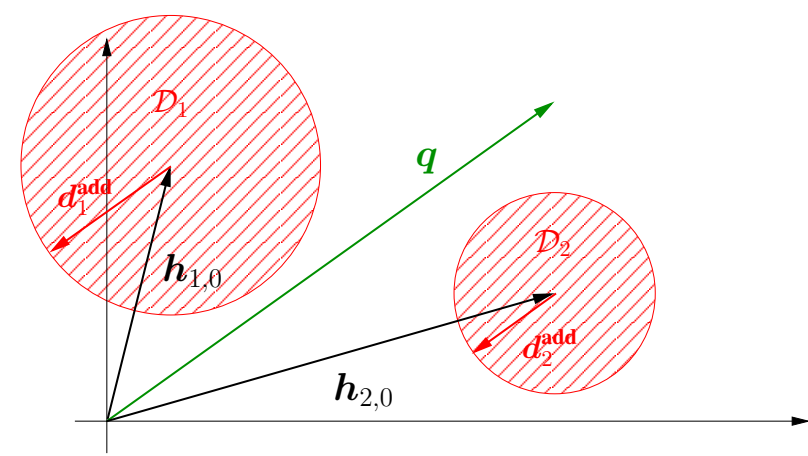

Fig. 4. Visualization of the influence of additive uncertainty for the MISO BBC. The worst-case perturbations $\boldsymbol{d}_{i}^{\text {add }}(\boldsymbol{Q})$ are anti-parallel to the direction of the transmit strategy $\boldsymbol{q}$.

which shows that the desired rates are actually achieved. This completes the proof.

Proposition 2 shows that for any given single beam transmit strategy $\boldsymbol{Q}=P \boldsymbol{u}_{\boldsymbol{q}} \boldsymbol{u}_{\boldsymbol{q}}^{H}$ the worst-case perturbations $\boldsymbol{d}_{i}^{\text {add }}(\boldsymbol{Q})$, $i=1,2$, are anti-parallel to the chosen transmit strategy $\boldsymbol{Q}=$ $P \boldsymbol{u}_{\boldsymbol{q}} \boldsymbol{u}_{\boldsymbol{q}}^{H}$. Figure 4 visualizes this behavior. Note that this in contrast to the SIMO case, where the worst-case perturbation is anti-parallel to the nominal channel.

Unfortunately, we run into the same problem as for the corresponding scenario under sent-site multiplicative channel uncertainty, cf. Section IV-B. The worst-case additive perturbation depends on the chosen transmit strategy while the optimal transmit strategy again depends on the actual perturbation. This makes it difficult to analyze the compound and worstcase capacity regions of the MISO BBC under additive channel uncertainty.

\section{CONCLUSION}

In this paper we studied bidirectional relaying under channel uncertainty. This required the analysis of the multiantenna bidirectional broadcast channel under multiplicative and additive channel uncertainty for which we analyzed the compound and worst-case capacity regions. Further, we characterized robust transmit strategies and identified worst-case perturbations.

Unfortunately, under additive channel uncertainty but also for some multiplicative uncertainty scenarios, the worst-case perturbations usually depend on the chosen transmit strategy. On the other hand the optimal transmit strategies also depend on the actual channel and therewith on the perturbations. This makes it difficult to characterize the corresponding compound and worst-case capacity regions in closed form, since they are determined by max min and min max optimization problems which involves optimizations over both, the transmit strategy and the perturbations.

Here we only analyzed the scenario where the relay helps to exchange the individual messages of both nodes. As future work it would be interesting and important to further analyze the scenario where the relay integrates additional services such as multicast or confidential services while suffering from uncertainty in channel state information. Physical layer service 
integration (PLSI) of bidirectional, common, and confidential services in multiantenna bidirectional relay networks is analyzed in [45] for perfect CSI.

\section{REFERENCES}

[1] B. Rankov and A. Wittneben, "Spectral Efficient Protocols for HalfDuplex Fading Relay Channels," IEEE J. Sel. Areas Commun., vol. 25, no. 2, pp. 379-389, Feb. 2007.

[2] P. Larsson, N. Johansson, and K.-E. Sunell, "Coded Bi-directional Relaying," in Proc. 5th Scandinavian Workshop on Ad Hoc Networks, Stockholm, Sweden, May 2005, pp. 851-855.

[3] Y. Wu, P. Chou, and S.-Y. Kung, "Information Exchange in Wireless Networks with Network Coding and Physical-Layer Broadcast," in Proc. Conf. Inf. Sciences and Systems, Baltimore, MD, USA, Mar. 2005, pp. $1-6$.

[4] R. Knopp, "Two-Way Radio Networks With a Star Topology," in Proc. Int. Zurich Seminar on Commun., Zurich, Switzerland, Feb. 2006, pp. 154-157.

[5] E. Biglieri, R. Calderbank, A. Constantinides, A. Goldsmith, A. Paulraj, and H. V. Poor, MIMO Wireless Communications. Cambridge University Press, 2007.

[6] T. J. Oechtering, C. Schnurr, I. Bjelaković, and H. Boche, "Broadcast Capacity Region of Two-Phase Bidirectional Relaying," IEEE Trans. Inf. Theory, vol. 54, no. 1, pp. 454-458, Jan. 2008.

[7] S. J. Kim, P. Mitran, and V. Tarokh, "Performance Bounds for Bidirectional Coded Cooperation Protocols," IEEE Trans. Inf. Theory, vol. 54, no. 11, pp. 5235-5241, Nov. 2008.

[8] G. Kramer and S. Shamai (Shitz), "Capacity for Classes of Broadcast Channels with Receiver Side Information," in Proc. IEEE Inf. Theory Workshop, Tahoe City, CA, USA, Sep. 2007, pp. 313-318.

[9] L.-L. Xie, "Network Coding and Random Binning for Multi-User Channels," in Proc. Canadian Workshop on Inf. Theory, Jun. 2007, pp. $85-88$.

[10] R. F. Wyrembelski, T. J. Oechtering, I. Bjelaković, C. Schnurr, and H. Boche, "Capacity of Gaussian MIMO Bidirectional Broadcast Channels," in Proc. IEEE Int. Symp. Inf. Theory, Toronto, Canada, Jul. 2008, pp. 584-588.

[11] T. J. Oechtering, R. F. Wyrembelski, and H. Boche, "Multiantenna Bidirectional Broadcast Channels - Optimal Transmit Strategies," IEEE Trans. Signal Process., vol. 57, no. 5, pp. 1948-1958, May 2009.

[12] T. J. Oechtering, E. A. Jorswieck, R. F. Wyrembelski, and H. Boche, "On the Optimal Transmit Strategy for the MIMO Bidirectional Broadcast Channel," IEEE Trans. Commun., vol. 57, no. 12, pp. 3817-3826, Dec. 2009.

[13] J. Zhao, M. Kuhn, A. Wittneben, and G. Bauch, "Optimum TimeDivision in MIMO Two-Way Decode-and-Forward Relaying Systems," in Proc. Asilomar Conf. Signals, Systems, Computers, Pacific Grove, CA, USA, Oct. 2008, pp. 1494-1500.

[14] A. Sezgin, M. A. Khajehnejad, A. S. Avestimehr, and B. Hassibi, "Approximate capacity region of the two-pair bidirectional Gaussian relay network," in Proc. IEEE Int. Symp. Inf. Theory, Seoul, Korea, Jun. 2009, pp. $2018-2022$.

[15] P. Popovski and T. Koike-Akino, Coded Bidirectional Relaying in Wireless Networks, ser. New Directions in Wireless Communications Research. Springer US, 2009, ch. 11, pp. 291-316.

[16] R. Zhang, Y.-C. Liang, C. C. Chai, and S. Cui, "Optimal Beamforming for Two-Way Multi-Antenna Relay Channel with Analogue Network Coding," IEEE J. Sel. Areas Commun., vol. 27, no. 5, pp. 699-712, Jun. 2009.

[17] H. Q. Ngo, T. Q. S. Quek, and H. Shin, "Amplify-and-Forward TwoWay Relay Networks: Error Exponents and Resource Allocation," IEEE Trans. Commun., vol. 58, no. 9, pp. 2653-2666, Sep. 2009.

[18] F. Roemer and M. Haardt, "Tensor-Based Channel Estimation and Iterative Refinements for Two-Way Relaying With Multiple Antennas and Spatial Reuse," IEEE Trans. Signal Process., vol. 58, no. 11, pp. 5720-5735, Nov. 2010

[19] E. Yilmaz, R. Zakhour, D. Gesbert, and R. Knopp, "Multi-pair Two-way Relay Channel with Multiple Antenna Relay Station," in Proc. IEEE Int. Conf. Commun., Cape Town, South Africa, May 2010, pp. 1-5.

[20] C. Schnurr, T. J. Oechtering, and S. Stańczak, "Achievable Rates for the Restricted Half-Duplex Two-Way Relay Channel," in Proc. Asilomar Conf. Signals, Systems, Computers, Pacific Grove, CA, USA, Nov. 2007, pp. 1468-1472.
[21] D. Gündüz, E. Tuncel, and J. Nayak, "Rate Regions for the Separated Two-Way Relay Channel," in Proc. Allerton Conf. Commun., Control, Computing, Sep. 2008, pp. 1333-1340.

[22] P. Zhong and M. Vu, "Compress-Forward without Wyner-Ziv Binning for the One-Way and Two-Way Relay Channels," in Proc. Allerton Conf. Commun., Control, Computing, Urbana-Champaign, IL, USA, Sep. 2011, pp. 426-433.

[23] L. Ong, C. M. Kellett, and S. J. Johnson, "Functional-Decode-Forward for the General Discrete Memoryless Two-Way Relay Channel," in Proc. IEEE Int. Conf. Commun. Systems, Singapore, Nov. 2010, pp. 351-355.

[24] M. P. Wilson, K. Narayanan, H. D. Pfister, and A. Sprintson, "Joint Physical Layer Coding and Network Coding for Bidirectional Relaying," IEEE Trans. Inf. Theory, vol. 56, no. 11, pp. 5641-5654, Nov. 2010.

[25] W. Nam, S.-Y. Chung, and Y. H. Lee, "Capacity of the Gaussian TwoWay Relay Channel to Within $\frac{1}{2}$ Bit," IEEE Trans. Inf. Theory, vol. 56, no. 11 , pp. $5488-5494$, Nov. 2010

[26] I.-J. Baik and S.-Y. Chung, "Network Coding for Two-Way Relay Channels using Lattices," Telecommunications review, vol. 17, no. 17, pp. 1009-1021, 2007.

[27] B. Nazer and M. Gastpar, "Compute-and-Forward: Harnessing Interference through Structured Codes," IEEE Trans. Inf. Theory, vol. 57, no. 10, pp. 6463-6486, Oct. 2011.

[28] Y. Song and N. Devroye, "A Lattice Compress-and-Forward Scheme," in Proc. IEEE Inf. Theory Workshop, Paraty, Brasil, Oct. 2011, pp. 110114.

[29] S. J. Kim, B. Smida, and N. Devroye, "Lattice Strategies for a Multi-Pair Bi-Directional Relay Network," in Proc. IEEE Int. Symp. Inf. Theory, Saint Petersburg, Russia, Jul. 2011, pp. 2243-2247.

[30] S. H. Lim, Y.-H. Kim, A. El Gamal, and S.-Y. Chung, "Layered Noisy Network Coding," in Proc. IEEE Wireless Network Coding Conf., Boston, MA, USA, Jun. 2010, pp. 1-6.

[31] — - "Noisy Network Coding," IEEE Trans. Inf. Theory, vol. 57, no. 5, pp. 3132-3152, May 2011.

[32] G. Kramer and J. Hou, "Short-Message Quantize-Forward Network Coding," in Proc. 8th Int. Workshop on Multi-Carrier Systems \& Solutions, Herrsching, Germany, May 2011, pp. 1-3.

[33] —_, "On Message Lengths for Noisy Network Coding," in Proc. IEEE Inf. Theory Workshop, Paraty, Brasil, Oct. 2011, pp. 430-431.

[34] N. Vucic and H. Boche, "Robust QoS-Constrained Optimization of Downlink Multiuser MISO Systems," IEEE Trans. Signal Process., vol. 57, no. 2, pp. 714-725, Feb. 2009.

[35] N. Vucic, H. Boche, and S. Shi, "Robust Transceiver Optimization in Downlink Multiuser MIMO Systems," IEEE Trans. Signal Process., vol. 57, no. 9, pp. 3576-3587, Sep. 2009.

[36] D. Blackwell, L. Breiman, and A. J. Thomasian, "The Capacity of a Class of Channels," Ann. Math. Stat., vol. 30, no. 4, pp. 1229-1241, Dec. 1959.

[37] J. Wolfowitz, "Simultaneous Channels," Arch. Rational Mech. Analysis, vol. 4, no. 4, pp. 371-386, 1960.

[38] W. L. Root and P. P. Varaiya, "Capacity of Classes of Gaussian Channels," SIAM J. Appl. Math, vol. 16, no. 6, pp. 1350-1393, 1968.

[39] D. P. Palomar, J. M. Cioffi, and M. A. Lagunas, "Uniform Power Allocation in MIMO Channels: A Game-Theoretic Approach," IEEE Trans. Inf. Theory, vol. 49, no. 7, pp. 1707-1727, Jul. 2003.

[40] S. Loyka and C. D. Charalambous, "On the Capacity of a Class of MIMO Channels Subject to Normed Uncertainty," in Proc. IEEE Int. Symp. Inf. Theory, Toronto, Canada, Jul. 2008, pp. 2578-2582.

[41] A. Wiesel, Y. C. Eldar, and S. Shamai (Shitz), "Beamforming Maximizes the Rank One Ricean MIMO Compound Capacity," in Proc. IEEE Signal Process. Adv. Wireless Commun., New York, NY, USA, Jun. 2005, pp. 323-327.

[42] - "Optimization of the MIMO Compound Capacity," IEEE Trans. Wireless Commun., vol. 6, no. 3, pp. 1094-1101, Mar. 2007.

[43] R. F. Wyrembelski, I. Bjelaković, T. J. Oechtering, and H. Boche, "Optimal Coding Strategies for Bidirectional Broadcast Channels under Channel Uncertainty," IEEE Trans. Commun., vol. 58, no. 10, pp. 2984 2994, Oct. 2010.

[44] R. A. Horn and C. R. Johnson, Matrix Analysis. Cambridge University Press, 1999.

[45] R. F. Wyrembelski and H. Boche, "Service Integration in Multiantenna Bidirectional Relay Networks: Public and Confidential Services," in Proc. IEEE Global Commun. Conf., Houston, TX, USA, Dec. 2011, pp. 914-918. 